\title{
Robust Variable-Scale Bilateral Control for Micro Teleoperation
}

\author{
Emmanuel Vander Poorten, Takahiro Kanno and Yasuyoshi Yokokohji
}

\begin{abstract}
This paper discusses variable-scale bilateral control for micro teleoperation. A method is proposed to synthesize robust variable-scale controllers that guarantee system stability and realize robust performance in contact with any arbitrary, but passive, operator and environment. The proposed method is based on the formulation as a robust output feedback constant scaled $\mathcal{H}_{\infty}$ problem. A variable scaled teleoperation system appears as a polytopic linear parameter-varying (LPV) plant, with an affine relation on the scale factors. Gain-scheduling is used to derive robust stable time-variant $\mathcal{H}_{\infty}$ controllers for online variable scaling. Improved controller performance is obtained through the introduction of an affine and scheduledparameter-dependent weighting. The controller synthesis problem is formulated as a set of linear matrix inequalities (LMI's) augmented with a nonconvex rank condition. An efficient algorithm is constructed to synthesize a set of sub-optimal controllers over the parameter space. The validity of the proposed methods are confirmed experimentally.
\end{abstract}

\section{INTRODUCTION}

Recently, scaled teleoperation is receiving an increased amount of attention within the teleoperation community. Especially the demands from maturing fields like microassembly or surgical robotics stimulate the renewed interest. The success of scaled teleoperation applications in these domains will depend on the usefulness and user-friendliness of these systems.

Indeed, intuitivity of use, sometimes described as 'telepresence' [1] or 'tele-existence' [2], which forms already a major issue in normal teleoperation, becomes an even more stringent requirement for scaled teleoperation. This is so because together with the scaling of forces and velocities also the feeling (impedance) of the remote environment is being distorted. Only some combinations of scale factors will result in an intuitive feeling. Note that these, typically taskdependent, scalings manage to capture only some physical phenomena occurring in the micro-world and translate them to the macro-(or human-scaled) world [3],[4]. Another reason follows from the fact that a certain set of scale factors also implies a specific choice for the trade-off that exists between the resolution and the range of operation in the micro-world. Also this choice benefits from a task-dependent answer.

When developing a flexible teleoperator to tackle various tasks, one might design a set of controllers for different scale factors and switch between them according to the task at hand. A first problem appearing here is the ease to loose

Emmanuel Vander Poorten was with the Department of Mechanical and Engineering and Science of Kyoto University. He is currently with the Department of Mechanical Engineering of the Catholic University of Leuven.manu.vanderpoorten@gmail.com

Takahiro Kanno and Yasuyoshi Yokokohji are with the Department of Mechanical Engineering and Science, Kyoto University. yokokohji@me.kyoto-u.ac.jp

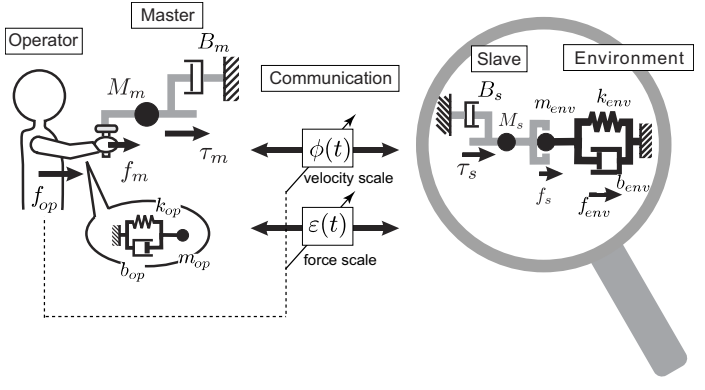

Fig. 1. Variable-scale 1-d.o.f. master-slave system

the 'big picture'. This problem also occurs in microscope visualization tasks; once something disappears from our view (or reach) in the magnified view, it becomes difficult to find it back. A second problem is that such a controller switch also requires the operator to make a 'mental switch'. As long as he/she is not fully accustomed to the new scale factors, his/her commands will divert from the intended ones. When controller switches occur e.g. during tissue manipulations, this could lead to tissue damage and so on.

This article advocates the use of online, continuously adaptable, user-commanded variable-scale teleoperation, presented schematically in Fig.1. The operator can determine at any moment which set of scale factors leads to intuitive manipulation (feeling, range, resolution) for a specific task, and let him/her adapt these scales swiftly to tackle the subsequent task. Also the effect of mental switches is less outspoken as changes are gradually and commanded by the operator self. Next to improvement in user-friendliness also increase in system flexility is expected. To see this, simply recall the parallel with pure visualization tasks; here, a variable-scale system would correspond to a modern camera with adaptable zoom lens, whereas a fixed-scale device would correspond to a classic camera with constant focal length.

Although the usefulness of variable-scale teleoperation might be clear, the fact is that such a system is timevariable and thus more complex to analyze and control. This is one of the main reasons why the vast majority of current scaled teleoperation research such as [3]-[5] treats fixed-scale systems. Just a few works discuss variable-scale teleoperation. Dubey [6] adapted velocity scale factors according to an offline determined, position-dependent scalepattern. Boukhnifer [7] developed task-based time-variable $\mathcal{H}_{\infty}$-based controllers which are robust against a varying time delay. Online adaptation of the scale factors is claimed, but it is unclear how this affects the stability of the approach. Sano [8] proposed gain scheduling to vary scale factors online. However, operator dynamics are not accounted for 
during the controller synthesis and also the remote environment is assumed to be known.

This paper introduces a method to develop variable-scale bilateral controllers for micro teleoperation. The controllers are designed to interact stably with any arbitrary passive operators and environments. Gain-scheduling is used to derive the time-variable controller as a function of the schedule parameters: the force scale $\varepsilon$ and the velocity scale $\phi$, belong to a prespecified domain $\mathcal{A}$ shown in Fig.2. An affine parameter-dependent weighting method is further introduced to allow for a better tuning of the system performance over the space of schedule parameters. Note that for simplicity this article focusses on one dimensional systems. Also time-delay is not treated here, although an expansion towards scheduling according to the amount of time delay is also possible [9].

The layout of this article is as follows. The basic equations of a master-slave system and the derivation of a generalized plant is done in section II. Section III explains gainscheduling for variable-scale teleoperation. A method to vary the weights of the different controller objectives is introduced in section IV. Then, experimental results are presented in section $\mathrm{V}$ and finally conclusions and directions for further work are sketched in section VI.

\section{FORMULATION OF SCALED TELEOPERATION IN $\mathcal{H}_{\infty}$ FRAMEWORK}

In this section some master-slave fundamentals are restated and a generalized plant for $\mathcal{H}_{\infty}$-control is constructed. For simplicity, the discussion is limited to a 1-d.o.f. master-slave system, presented schematically in Fig.1.

\section{A. Master-slave fundamentals}

Master and slave are modeled by simple mass-dampers:

$$
\begin{aligned}
u_{m}(t)+f_{m}(t) & =M_{m} \ddot{x}_{m}(t)+B_{m} \dot{x}_{m}(t), \\
u_{s}(t)-f_{s}(t) & =M_{s} \ddot{x}_{s}(t)+B_{s} \dot{x}_{s}(t),
\end{aligned}
$$

with $u_{m}(t)$ and $u_{s}(t)$ the control inputs of master and slave, $f_{m}(t)$ and $f_{s}(t)$ are the force exerted by the operator on the master and by the slave on the environment. Mass and damping are $M_{m}, B_{m}, M_{s}$ and $B_{s}$. The velocity and acceleration of master and slave are $\dot{x}_{m}, \ddot{x}_{m}, \dot{x}_{s}$ and $\ddot{x}_{s}$.

The operator and the environment dynamics are typically nonlinear and time-variant. The only assumption made in this work, and which was also made by Hogan [10], is that

ASSUMPTION 1: the operator and the environment can be treated as passive elements and they do not generate a force that causes the system to become unstable.

However, for notational convenience they are sometimes described as LTI-systems in this paper, in such case:

$$
\begin{aligned}
f_{o p}(t)-f_{m}(t) & =m_{o p} \ddot{x}_{m}(t)+b_{o p} \dot{x}_{m}(t)+k_{o p} x_{m}(t), \\
f_{\text {env }}(t)+f_{s}(t) & =m_{\text {env }} \ddot{x}_{s}(t)+b_{\text {env }} \dot{x}_{s}(t)+k_{\text {env }} x_{s}(t),(4)
\end{aligned}
$$

holds, where the operator's arm and the environment are modeled as mass-spring-dampers with $m_{o p}, b_{o p}, k_{o p}, m_{e n v}$, $b_{e n v}$ and $k_{e n v}$. The forces $f_{o p}(t)$ and $f_{e n v}(t)$ are then exogenous forces realized by the operator's muscles and an active element at the remote site.

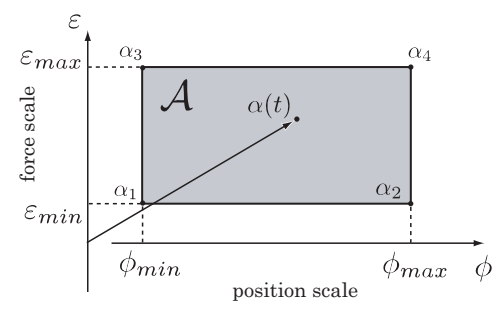

Fig. 2. Variable scale factors within prespecified domain

\section{B. Ideal response}

We define the ideal response of variable-scale teleoperation as 'ideal scaled kinesthetic coupling':

$$
\begin{aligned}
\dot{x}_{m}(t) & =\phi(t) \dot{x}_{s}(t), \\
f_{m}(t) & =\varepsilon(t) f_{s}(t),
\end{aligned}
$$

for time variable scale factors $\boldsymbol{\alpha}(t)=[\phi(t), \varepsilon(t)]^{T}$. Note, that the time variable nature of $\phi(t)$ makes that there is no fixed relation between master and slave position. Instead

$$
x_{m}(t)=\hat{x}_{s}(t)
$$

should hold (ideally), for $\hat{x}_{s}(t) \triangleq \int_{0}^{t} \phi(\tau) \dot{x}_{s}(\tau) d \tau$. Also note that in this work we assume that the vector of scale factors $\boldsymbol{\alpha}(t)=[\phi(t), \varepsilon(t)]^{T}$ belongs to a closed convex set $\mathcal{A}=$ $\left\{(\phi, \varepsilon) \mid \phi_{\min } \leq \phi \leq \phi_{\max }, \varepsilon_{\min } \leq \varepsilon \leq \varepsilon_{\max }\right\}$, shown in Fig.2. On the other hand, no assumptions are made on the rate of variation of $\boldsymbol{\alpha}(t)$.

\section{Coupled stability}

A good controller should achieve ideal scaled kinesthetic coupling as close as possible, but should at the same time guarantee the stability of the whole system. Since the stability is not only a function of the teleoperator, but also depends on the operator and environment, so-called 'coupled stability' should be considered.

Colgate formulated a condition for coupled stability [3] based on the reformulation of the different elements towards their scattering form, as shown in Fig.3. The scattering matrix of the master-slave system $\boldsymbol{S}_{m s s}$ is connected to the operator and the environment, $\boldsymbol{S}_{o p}$ and $\boldsymbol{S}_{e n v}$, under the form of a structured uncertainty block $\Delta_{o e}$. Note that for passive operator and environment $\left\|\boldsymbol{S}_{o p}\right\|_{\infty} \leq 1$ and $\left\|\boldsymbol{S}_{e n v}\right\|_{\infty} \leq 1$ holds, so that $\left\|\Delta_{o e}\right\|_{\infty} \leq 1$. Colgate's condition for coupled stability is then that, for $\boldsymbol{S}_{m s s}(s)$ analytic in $\Re(s) \geq 0$,

$$
\mu_{\boldsymbol{\Delta}_{o e}}\left(\boldsymbol{S}_{m s s}(s)\right) \leq 1, \forall \omega,
$$

where $\mu_{\boldsymbol{\Delta}_{o e}}\left(\boldsymbol{S}_{m s s}(s)\right)$ is the structured singular value[11] of the system $\boldsymbol{S}_{m s s}(s)$ against the structured uncertainty block $\boldsymbol{\Delta}_{o e}$. Note, that in the case of (one dimensional) teleoperation, (8) reduces to:

$$
\inf _{\boldsymbol{D}} \bar{\sigma}\left(\boldsymbol{D} \boldsymbol{S}_{m s s} \boldsymbol{D}^{-1}\right) \leq 1,
$$

for a block diagonal matrix $\boldsymbol{D}=\operatorname{diag}\left(\left[d_{1}(s), d_{2}(s)\right]\right)$ which has a structure similar to $\boldsymbol{\Delta}_{o e}$ with positive real frequency dependent variables $d_{1}(s), d_{2}(s)$. 


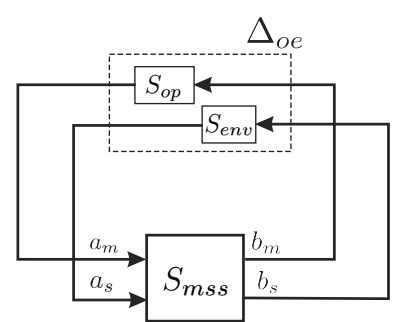

Fig. 3. Operator/environment as structured uncertainty

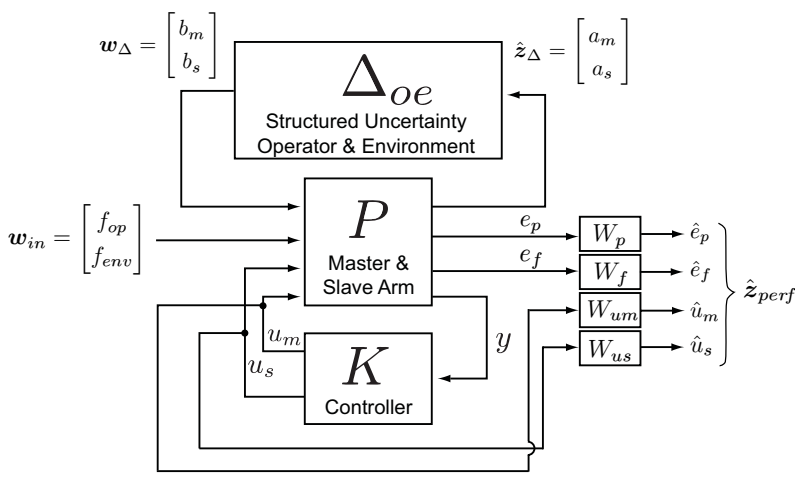

Fig. 4. Generalized plant of a master-slave system

However, above condition provides only a necessary and sufficient condition for stability in contact with arbitrary passive linear time-invariant (LTI) operators and environments. As practical operators and environments are typically nonlinear and/or time-variable, in this work, following theorem [3] is adhered to:

THEOREM 1 (Robust stability): A necessary and sufficient condition for the stability of a master-slave system interacting with any arbitrary non-linear and time-variant operator and environment is that the scattering matrix $\boldsymbol{S}_{m s s}(s)$ is analytic in $\Re(s) \geq 0$ and that the scaled $\mathcal{H}_{\infty}$ norm

$$
\inf _{\boldsymbol{D}} \bar{\sigma}\left(\boldsymbol{D} \boldsymbol{S}_{m s s} \boldsymbol{D}^{-1}\right) \leq 1
$$

where the minimization happens over all matrices $\boldsymbol{D}=$ $\operatorname{diag}\left(\left[d_{1}, d_{2}\right]\right)$ with a structure similar to that of the uncertainty block $\boldsymbol{\Delta}_{o e}$ and frequency-independent $d_{1}$ and $d_{2}$.

\section{Generalized plant for variable-scale teleoperation}

Although the reorganization of operator and environment as a structured uncertainty is originally developed to analyze the stability of a teleoperation system[3], it proves to be equally useful to synthesize $\mathcal{H}_{\infty}$ based coupled stable teleoperation controllers. Indeed, Fig. 3 can be rewritten easily towards a typical $\mathcal{H}_{\infty}$ structure of Fig.4, where the bounded uncertainty block $\left\|\boldsymbol{\Delta}_{o e}\right\|_{\infty} \leq 1$ captures the interaction with passive operator and environment. A $\mathcal{H}_{\infty}$ controller that stabilizes $P$ with respect to any $\left\|\boldsymbol{\Delta}_{o e}\right\| \leq 1$ makes the controlled plant 'robust stable' for every contact with passive operator and environment. Note that this observation was already made by Yoshikawa et al. [12] (only in Japanese).
To achieve the ideal response discussed in section II-B, the control objectives can be specified as:

$$
\boldsymbol{z}_{\text {perf }}=\left[\begin{array}{c}
e_{p} \\
e_{f} \\
u_{m} \\
u_{s}
\end{array}\right] \triangleq\left[\begin{array}{c}
\rho\left(x_{m}(t)-\hat{x}_{s}(t)\right)+\left(\dot{x}_{m}(t)-\phi(t) \dot{x}_{s}(t)\right) \\
f_{m}(t)-\varepsilon(t) f_{s}(t) \\
u_{m} \\
u_{s}
\end{array}\right],
$$

where $\rho$ denotes a constant weighting for the position error relative to the velocity error. The last two elements in (11), $u_{m}$ and $u_{s}$, prevent the use of excessive control effort.

After some algebraic manipulations can the system (1), (2), (3), (4), be transformed to its scattering form. Then, the total system, together with the control objectives (11), can be written as the following generalized plant $P$ for variablescaled teleoperation:

$$
P(\boldsymbol{\alpha}):\left\{\begin{array}{l}
\dot{\boldsymbol{x}}=\boldsymbol{A}(\phi) \boldsymbol{x}+\boldsymbol{B}_{1} \boldsymbol{w}+\boldsymbol{B}_{2} \boldsymbol{u} \\
\boldsymbol{z}=\boldsymbol{C}_{1}(\phi, \varepsilon) \boldsymbol{x}+\boldsymbol{D}_{11}(\varepsilon) \boldsymbol{w}+\boldsymbol{D}_{12} \boldsymbol{u} . \\
\boldsymbol{y}=\boldsymbol{C}_{2} \boldsymbol{x}+\boldsymbol{D}_{21} \boldsymbol{w}
\end{array} .\right.
$$

Considering space limitations, the elements of matrices $\boldsymbol{A}$, $\boldsymbol{B}_{1}, \boldsymbol{B}_{2}, \boldsymbol{C}_{1}, \boldsymbol{C}_{2}, \boldsymbol{D}_{11}, \boldsymbol{D}_{12}$ and $\boldsymbol{D}_{21}$ are not described in detail here. For the details, please refer to [13]. One important point here is that the scale factors actually appear in an affine way in the matrices $\boldsymbol{A}, \boldsymbol{C}_{1}$ and $\boldsymbol{D}_{11}$. Above discussion is now developed further to describe robust performance.

\section{E. Robust performance for scaled teleoperation}

To specify the relative importance of the different and competing control objectives in (11), frequency-dependent weightings: $\boldsymbol{W}=\operatorname{diag}\left(\left[W_{p}, W_{f}, W_{u m}, W_{u s}\right]\right)$ are added as shown in Fig.4. The weighted form of signal $x$ is written as $\hat{x}$. System $P(\boldsymbol{\alpha})$ augmented with weights $\boldsymbol{W}$ is written as:

$$
\hat{P}(\boldsymbol{\alpha}):\left\{\begin{array}{l}
\dot{\hat{\boldsymbol{x}}}=\hat{\boldsymbol{A}}(\phi) \hat{\boldsymbol{x}}+\hat{\boldsymbol{B}}_{1} \boldsymbol{w}+\hat{\boldsymbol{B}}_{2} \boldsymbol{u} \\
\hat{\boldsymbol{z}}=\hat{\boldsymbol{C}}_{1}(\phi, \varepsilon) \hat{\boldsymbol{x}}+\hat{\boldsymbol{D}}_{11}(\varepsilon) \boldsymbol{w}+\hat{\boldsymbol{D}}_{12} \boldsymbol{u} . \\
\boldsymbol{y}=\hat{\boldsymbol{C}}_{2} \hat{\boldsymbol{x}}+\hat{\boldsymbol{D}}_{21} \boldsymbol{w}
\end{array}\right.
$$

Where $\hat{\boldsymbol{x}}=\left[\boldsymbol{x}, \boldsymbol{x}_{w}\right]^{T}$ contains the states of the generalized plant $\boldsymbol{x}$ and of the weighting filters $\boldsymbol{x}_{w}$. Now, the controlled and weighted system $P_{a u g}$, containing the generalized plant $P$ and the weighting filters $W$, together with the controller $K$ with state space representation:

$$
K(\boldsymbol{\alpha}):\left\{\begin{array}{l}
\dot{\boldsymbol{x}}_{K}=\boldsymbol{A}_{K}(\boldsymbol{\alpha}) \boldsymbol{x}_{K}+\boldsymbol{B}_{K}(\boldsymbol{\alpha}) \boldsymbol{y} \\
\boldsymbol{u}=\boldsymbol{C}_{K}(\boldsymbol{\alpha}) \boldsymbol{x}_{K}+\boldsymbol{D}_{K}(\boldsymbol{\alpha}) \boldsymbol{y}
\end{array},\right.
$$

with controller state $\boldsymbol{x}_{K}$, is called 'robust performant', with user-defined performance $\gamma$, if $K$ can be designed so that $\left\|\boldsymbol{T}_{\text {perf }}\right\|_{\infty} \leq \gamma$ holds, where $\boldsymbol{T}_{\text {perf }}$ is the matrix of transfer functions from $\boldsymbol{w}_{\text {in }}=\left[f_{o p}, f_{\text {env }}\right]^{T}$ to $\hat{\boldsymbol{z}}_{\text {perf }}$. This problem can be reformulated as a robust stability problem against an augmented uncertainty block:

$\boldsymbol{\Delta}_{a u g} \triangleq\left\{\left[\begin{array}{cc}\boldsymbol{\Delta}_{o e} & 0 \\ 0 & \boldsymbol{\Delta}_{\text {perf }}\end{array}\right]: \boldsymbol{\Delta}_{\text {perf }} \in \mathbb{C}^{2 \times 4}\right.$ and $\left.\left\|\boldsymbol{\Delta}_{\text {perf }}\right\|_{\infty} \leq 1\right\}$,

by closing a loop between $\boldsymbol{z}_{\text {perf }}$ and $\boldsymbol{w}_{\text {in }}$ over an additional uncertainty block $\boldsymbol{\Delta}_{\text {perf }}$ and by inserting the gain matrices

$$
\boldsymbol{\Gamma}_{l}=\left[\begin{array}{cc}
\boldsymbol{I}_{2 \times 2} & \mathbf{0}_{2 \times 4} \\
\mathbf{0}_{4 \times 2} & \frac{1}{\gamma^{\frac{1}{2}}} \boldsymbol{I}_{4 \times 4}
\end{array}\right], \quad \boldsymbol{\Gamma}_{r}=\left[\begin{array}{cc}
\boldsymbol{I}_{2 \times 2} & \mathbf{0}_{2 \times 2} \\
\mathbf{0}_{2 \times 2} & \frac{1}{\gamma^{\frac{1}{2}}} \boldsymbol{I}_{2 \times 2}
\end{array}\right]
$$




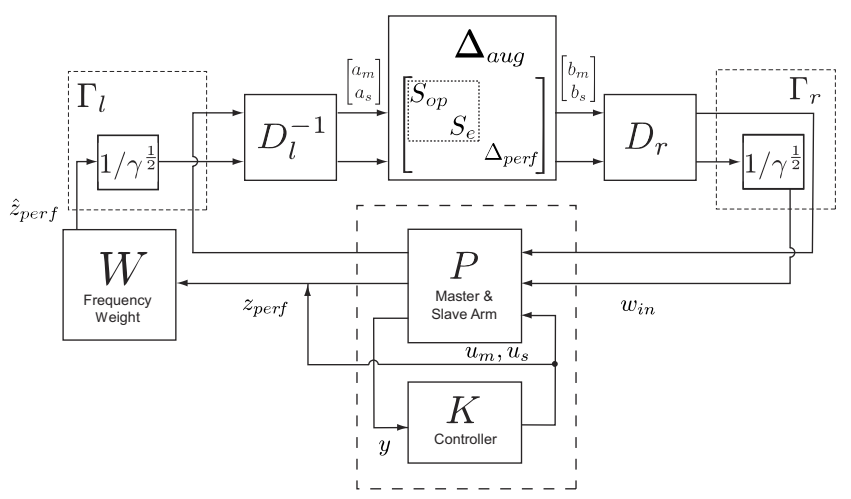

Fig. 5. Robust performance arrangement of a master-slave system

in the closed loop, as shown in Fig.5. To take full advantage of the structuredness of $\boldsymbol{\Delta}_{o e}$, rather than normal $\mathcal{H}_{\infty}$, constant scaled $\mathcal{H}_{\infty}$ (in line with Theorem 1) is proposed here. To this end, scaling matrices $\boldsymbol{D}_{l}^{-1}$ and $\boldsymbol{D}_{r}$, where

$$
\boldsymbol{D}_{l}=\left[\begin{array}{ccc}
d_{1} & 0 & \mathbf{0}_{1 \times 4} \\
0 & d_{2} & \mathbf{0}_{1 \times 4} \\
\mathbf{0}_{4 \times 1} & \mathbf{0}_{4 \times 1} & d_{3} \boldsymbol{I}_{4 \times 4}
\end{array}\right], \boldsymbol{D}_{r}=\left[\begin{array}{ccc}
d_{1} & 0 & \mathbf{0}_{1 \times 2} \\
0 & d_{2} & \mathbf{0}_{1 \times 2} \\
\mathbf{0}_{2 \times 1} & \mathbf{0}_{2 \times 1} & d_{3} \boldsymbol{I}_{2 \times 2}
\end{array}\right],
$$

with real and frequency-independent scale factors $d_{i} \in$ $\Re_{+}(i=1,2,3)$ are added. The scaled $\mathcal{H}_{\infty}$ synthesis uses the freedom in $d_{i}$ to come up with less conservative $\mathcal{H}_{\infty}$ controllers. Finally, all the different elements are gathered in Fig.5 and following theorem is constructed:

THEOREM 2 (Robust performance): A necessary and sufficient condition for $\gamma$-performance of a master-slave system whose input/output relations are described by the transfer function $\boldsymbol{T}_{\text {perf }}$, in contact with any non-linear and time variable operator and environment is that $\boldsymbol{T}_{\text {perf }}$ is analytic in $\operatorname{Re}(s) \geq 0$ and the scaled $\mathcal{H}_{\infty}$ norm satisfies

$$
\left\|\boldsymbol{D}_{r} \boldsymbol{\Gamma}_{r} \boldsymbol{T}_{p e r f} \boldsymbol{\Gamma}_{l} \boldsymbol{D}_{l}^{-1}\right\|_{\infty}<1 .
$$

The solution to the constant scaled $\mathcal{H}_{\infty}$ problem, summarized by (18) can now be reformulated as a set LMI's augmented with some matrix rank conditions (see Appendix A). The solution to these constraints with minimal $\gamma$ will lead to a robust controller with sub-optimal performance.

While the LMI's are convex constraints and efficient algorithms exist to solve them [14], the rank condition and the minimization of $\gamma$ are nonconvex. Fortunately, in the design of reduced order controllers, similar conditions also arise and quite a few heuristics have been proposed to derive local solutions to this problem (e.g. [15],[16] and [17]).

In this article, the cone complementarity linearization algorithm [15] by El Ghaoui is used to solve the nonconvex rank condition. It is a coordinate descent scheme that alternatively and iteratively fixes parts of the coordinates of the solution, while optimizing the remaining parameters. Because each intermediate step is itself a convex LMI program, it can be readily implemented. Although global convergence is not guaranteed, in practice good and fast convergence occurs. The local controller (14) for a specific $\alpha$ can be calculated from the found solution.

\section{VARIABLE-SCALE TELEOPERATION BY GAIN SCHEDULING}

\section{A. Derivation of a gain scheduling controller}

The minimization of $\gamma$ under the LMI's and rank condition constraints requires substantial calculations. The realization of variable-scale teleoperation by solving this problem for every possible combination of scale factors (Fig.2) is a nontractable problem. Even if all controllers were calculated offline and switched online as fuctions of $\boldsymbol{\alpha}(t)$, stability cannot be guaranteed. Such method might only work for sufficiently slow variations of $\boldsymbol{\alpha}(t)$ [18].

The observation that the generalized plant (12) has a linear parameter-varying (LPV) dependence, with state-space equations of the form:

$$
P(\boldsymbol{p})=\left\{\begin{array}{l}
\dot{\boldsymbol{x}}=\boldsymbol{A}(\boldsymbol{p}(t)) \boldsymbol{x}+\boldsymbol{B}_{1}(\boldsymbol{p}(t)) \boldsymbol{w}+\boldsymbol{B}_{2}(\boldsymbol{p}(t)) \boldsymbol{u} \\
\boldsymbol{z}=\boldsymbol{C}_{1}(\boldsymbol{p}(t)) \boldsymbol{x}+\boldsymbol{D}_{11}(\boldsymbol{p}(t)) \boldsymbol{w}+\boldsymbol{D}_{12}(\boldsymbol{p}(t)) \boldsymbol{u} \\
\boldsymbol{y}=\boldsymbol{C}_{2}(\boldsymbol{p}(t)) \boldsymbol{x}+\boldsymbol{D}_{21}(\boldsymbol{p}(t)) \boldsymbol{w}+\boldsymbol{D}_{22}(\boldsymbol{p}(t)) \boldsymbol{u}
\end{array}\right.
$$

on the time-varying parameters $\boldsymbol{p}(t)$, is a crucial insight that leads to the tractability of variable-scale teleoperation.

THEOREM 3 (Variable-scale teleop. as polytopic LPV): A variable-scale teleoperation system described by (12), where the scale factors may vary over the polytope $\mathcal{A}:\left\{[\phi, \varepsilon]^{T}: \phi \in\left\{\phi_{\min }, \phi_{\max }\right\}, \varepsilon \in\left\{\varepsilon_{\min }, \varepsilon_{\max }\right\}\right\}$ depicted in Fig.2, is a polytopic LPV system as (19), for which the scale factors $\boldsymbol{\alpha}(t)$ are treated as the schedule parameters $\boldsymbol{p}(t)$, varying over polytope $\mathcal{P}=\mathcal{A}$.

Note that also the weighted and controlled plant $P_{a u g}$ is a polytopic LPV system. Now, Apkarian[19] showed that the Bounded Real Lemma for a polytopic LPV system is valid within each element of a polytope of the parameter space if it holds at every vertex of the polytope, the so-called vertex property:

LEMMA 1 (Vertex property): LPV systems, such as (19), are stable with quadratic $\mathcal{H}_{\infty}$ performance $\gamma$ so that $\|\boldsymbol{y}\|_{2}<$ $\gamma\|\boldsymbol{u}\|_{2}$ along all possible trajectories of $\boldsymbol{p}$, if there exist a single matrix $\boldsymbol{X}>0$ so that

$$
\left(\begin{array}{ccc}
\boldsymbol{A}^{i^{T}} \boldsymbol{X}+\boldsymbol{X} \boldsymbol{A}^{i} & \boldsymbol{X} \boldsymbol{B}^{i} & \boldsymbol{C}^{i^{T}} \\
\boldsymbol{B}^{i^{T}} \boldsymbol{X} & -\gamma \boldsymbol{I} & \boldsymbol{D}^{i^{T}} \\
\boldsymbol{C}^{i} & \boldsymbol{D}^{i} & -\gamma \boldsymbol{I}
\end{array}\right)<\mathbf{0}
$$

holds for every vertex $i$ of the polytope $\mathcal{P}$.

Therefore, robust performance (18) of the master-slave system can be guaranteed over the whole range of scale factors if one can find a suboptimal solution to the $2^{n}$ sets of LMI's for $P_{\text {aug }}$ (in this work $n=2$ ). The existence of a single matrix solution $\boldsymbol{X}>0$ reflects the existence of one fixed Lyapunov function over the whole range of scale factors. In such case, the controlled system is stable for any $\dot{\boldsymbol{p}}(t)$. This might be conservative in situations where knowledge on $\dot{\boldsymbol{p}}(t)$ is available [20]. Since no assumptions on $\dot{\boldsymbol{p}}(t)$ are made in this work, arbitrary fast variations of $\boldsymbol{\alpha}(t)$ are allowed.

Now, the following result shows how controllers with quadratic $\mathcal{H}_{\infty}$ performance can be calculated efficiently [19]: 


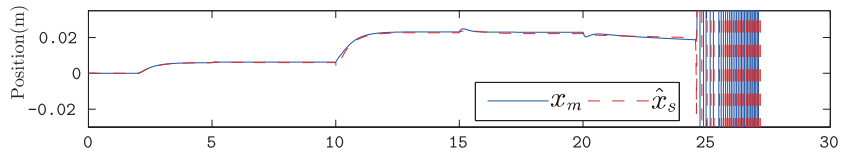

$\theta$
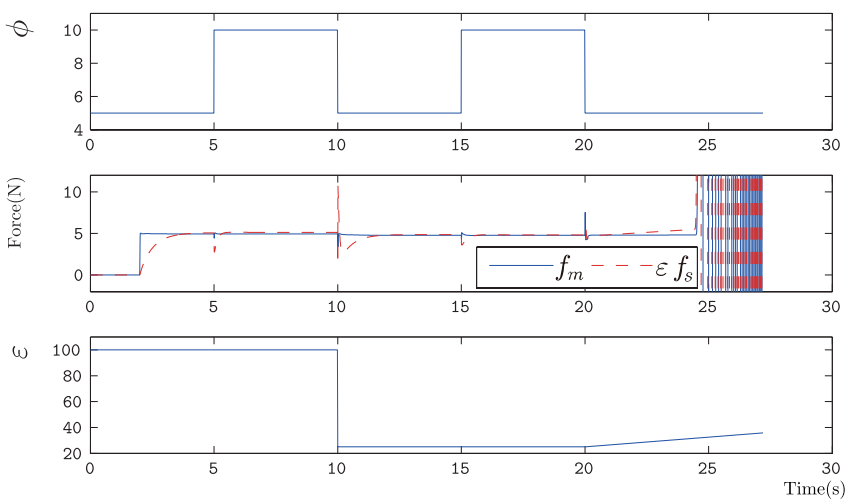

Fig. 6. Simulation result: Interpolation of fixed-scale controllers

LEMMA 2 (Quadratic $\mathcal{H}_{\infty}$ performance controllers):

For the polytopic plant of (19) and under the conditions that

(i) $\boldsymbol{D}_{22}(\boldsymbol{p})=\mathbf{0}$,

(ii) $\boldsymbol{B}_{2}(\boldsymbol{p}), \boldsymbol{C}_{2}(\boldsymbol{p}), \boldsymbol{D}_{12}(\boldsymbol{p})$ and $\boldsymbol{D}_{21}(\boldsymbol{p})$ are parameterindependent, and

(iii) the pairs $\left(\boldsymbol{A}(\boldsymbol{p}), \boldsymbol{B}_{2}\right)$ and $\left(\boldsymbol{A}(\boldsymbol{p}), \boldsymbol{C}_{2}\right)$ are quadratically stabilizable and quadratically detectable over $P$,

the polytopic controllers with state-space matrices $\boldsymbol{A}_{K}(\boldsymbol{p})$, $\boldsymbol{B}_{K}(\boldsymbol{p}), \boldsymbol{C}_{K}(\boldsymbol{p})$ and $\boldsymbol{D}_{K}(\boldsymbol{p})$ and given by :

$$
\left(\begin{array}{ll}
\boldsymbol{A}_{K}(\boldsymbol{p}) & \boldsymbol{B}_{K}(\boldsymbol{p}) \\
\boldsymbol{C}_{K}(\boldsymbol{p}) & \boldsymbol{D}_{K}(\boldsymbol{p})
\end{array}\right) \triangleq \sum_{i=1}^{2^{n}} a_{i} \Omega_{i}=\sum_{i=1}^{2^{n}} a_{i}\left(\begin{array}{ll}
\boldsymbol{A}_{K}^{i} & \boldsymbol{B}_{K}^{i} \\
\boldsymbol{C}_{K}^{i} & \boldsymbol{D}_{K}^{i}
\end{array}\right)
$$

give the closed-loop system a quadratic $\mathcal{H}_{\infty}$ performance with bound $\gamma$, if a single matrix $\boldsymbol{X}>0$ exists so that (20) holds for every vertex of $\mathcal{P}$.

If such $\boldsymbol{X}>0$ can be found, a possible choice of the LPV controller is then the polytopic controller given by (21), where the values $\left(a_{1}, \ldots, a_{2^{n}}\right)$ are the solution to the convex decomposition problem :

$$
\boldsymbol{p}=\sum_{i=1}^{2^{n}} a_{i} \boldsymbol{p}_{i}
$$

After calculating matrices $\boldsymbol{A}_{K}^{i}, \boldsymbol{B}_{K}^{i}, \boldsymbol{C}_{K}^{i}$ and $\boldsymbol{D}_{K}^{i}$, it suffices to solve (22) based on the measured values of the schedule parameter $\boldsymbol{p}(t) \in \mathcal{P}$ which is thus $\boldsymbol{\alpha}(t) \in \mathcal{A}$ in our case.

\section{B. Gain scheduling vs. locally interpolated controllers}

The derivation of a gain scheduling controller involves the solution of a large LMI containing the LMI conditions for every vertex of the parameter box. To avoid such computational cost, one might be tempted to employ a naive solution where the controller is obtained by deriving four fixed-scale controllers at each vertex of the parameter box and interpolate them similar to (21). This naive solution is much easier to obtain but cannot guarantee the stability under the variable scaling scenario.

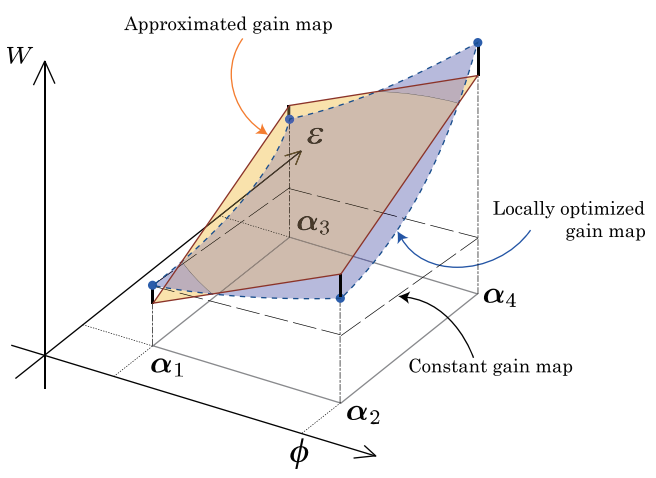

Fig. 7. Parameter dependent weight $W$

Fig.6 illustrates a simulation of such a naive controller. Since the space is limited, the parameters used in the simulation are not given here. But one can see clearly that this naive controller works well locally at each vertex. Also switching between controllers does not affect the stability. But when the scaling parameters are adapted continuously inside the parameter box, instability appears. From this simulation result, the need for the gain scheduling approach for the variable scaling scenario is clear.

\section{IMPROVING PERFORMANCE THROUGH USE OF PARAMETER-DEPENDENT WEIGHTINGS}

It is well-known that the performance of standard $\mathcal{H}_{\infty}$ controllers depends heavily on the quality of the selected weighting filters. One should also note that the dynamics of a system under scaled teleoperation vary greatly as the scale factor changes. Thus, one can expect that controllers designed for a certain constant set of weights $W$ show only good performance over a limited region of the parameter space. Therefore, we propose to use parameter-dependent weighting functions:

$$
W(\boldsymbol{\alpha}):\left\{\begin{array}{l}
\dot{\boldsymbol{x}}_{w}=\boldsymbol{A}_{w}(\boldsymbol{\alpha}) \boldsymbol{x}_{w}+\boldsymbol{B}_{w}(\boldsymbol{\alpha}) \boldsymbol{u}_{w} \\
\boldsymbol{y}_{w}=\boldsymbol{C}_{w}(\boldsymbol{\alpha}) \boldsymbol{x}_{w}+\boldsymbol{D}_{w}(\boldsymbol{\alpha}) \boldsymbol{u}_{w}
\end{array} .\right.
$$

This extra freedom is used to obtain a better performance over the whole parameter space. However, care should be taken not to violate the affine dependence of $P_{a u g}$ on $\boldsymbol{\alpha}$. For that reason, only the weights $W_{p}$ and $W_{f}$ can be made parameter-dependent. Whereas the other weights $W_{u m}$ and $W_{u s}$ must be kept parameter-independent.

Assume a set of 'optimal' weighting filters found through optimization of local fixed-scale controllers, depicted in Fig.7. Then, the parameter-dependent weightings are designed according to following heuristic:

ALGORITHM 1 (Scale-dependent weighting): The design of the weighting filters happens by solving a search for locally optimal weight factors (controllers) in at least three non-collinear locations of the parameter space. Then, a planar 'Approximated gain map' is constructed, minimizing the distance to the obtained local gains in a least-square sense. Finally, the weights of the approximated gain map, in the different vertices of the parameters space are calculated. 
The planarity of the Approximated gain map is a first condition to maintain the affine dependence on $P_{a u g}$. Secondly, the state space matrices of the filters must be designed so that all the terms $\boldsymbol{B}_{w}(\boldsymbol{\alpha}) \boldsymbol{C}_{1}(\boldsymbol{\alpha}), \boldsymbol{B}_{w}(\boldsymbol{\alpha}) \boldsymbol{D}_{11}(\boldsymbol{\alpha}), \boldsymbol{D}_{w}(\boldsymbol{\alpha}) \boldsymbol{C}_{1}(\boldsymbol{\alpha})$ and $\boldsymbol{D}_{w}(\boldsymbol{\alpha}) \boldsymbol{D}_{11}(\boldsymbol{\alpha})$, which appear in $\hat{P}(\alpha)$ (13) with parameterdependent weights, written in full as:

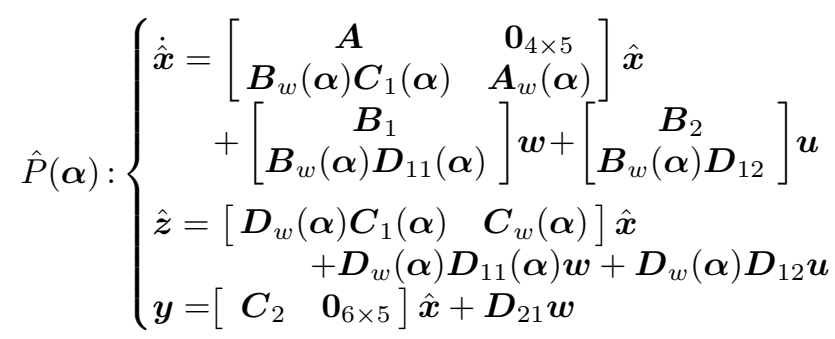

remain affine in the scale parameters. A smart choice of the state space representation of the filters must be done to preserve the affinity (see [13] for more details).

Now, the number and the locations where optimal local weights are calculated are in fact design parameters. Further research should be done to develop a solid strategy to optimize this selection procedure. In this work 4 optimal gains $W_{i}(i=1, \ldots, 4)$ were calculated for the 4 vertices $\boldsymbol{\alpha}_{i}(i=1, \ldots, 4)$. At present, only variations of gains of weighting filters are being investigated, but also variations in the locations of poles and zeros might prove useful.

The proposed method is also beneficial in the sense that it drastically reduces the computation time. Since the calculation of one variable-scale $\mathcal{H}_{\infty}$ based controller takes about 42 minutes on a Pentium $I V, 3.4 \mathrm{GHz}$ processor, using MATLAB's LMI toolbox [14], it is clear that 'blindly' deciding a combination of 6 weighting filters (for $W_{p}$ and $W_{f}$ at three locations) will require a large amount of iterations and a high computation time $(42 \times N$ minutes, where $N \gg 1)$. Indeed, many selections will fail to result in controllers with sufficient performance. The calculation of a local controller, on the other hand, only requires about 3 minutes. Therefore the proposed heuristic requires $\sum_{i=1}^{4} M_{i} \times 3+42$ minutes for obtaining locally optimized controllers at 4 vertices and a single run of calculation over the whole parameter space. Note that $M_{i}, i=1, \ldots, 4$ is much less than $N$ because only 2 weighting filters must be tuned towards a local optimum.

\section{EXPERIMENTAL RESULTS}

\section{A. The experimental setup}

The validity of the proposed method was experimentally examined on a 1-d.o.f. setup. The setup consists of a directdrive DC motor SCARA type of 3-d.o.f. master, shown in Fig.8. For the experiment only the elbow joint was actuated. The shoulder joint was mechanically fixed, whereas the wrist joint was left free to rotate. The link length of the actuated arm is $30 \mathrm{~cm}$. A six-axis force/torque sensor is mounted at the tip of the master. The position of the elbow joint is measured by encoder signals (120,000 pulses/rev.) and the velocity is measured by a combination of the counts of crystal clock pulses passed between every encoder pulse

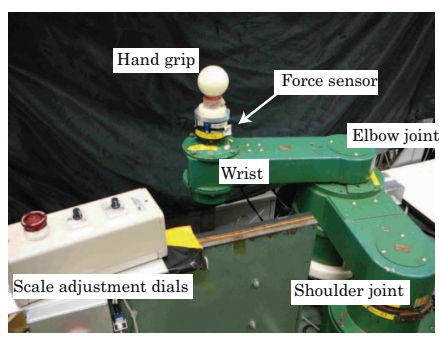

Fig. 8. Master arm

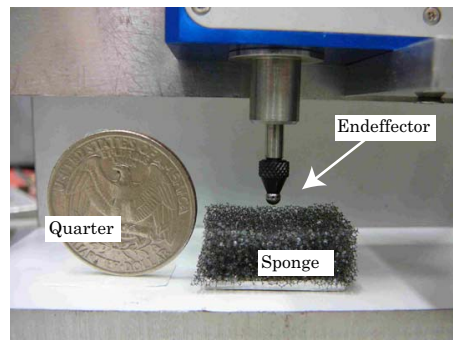

Fig. 9. Slave arm and spongy environment

TABLE I

CONTROLLER PARAMETERS FOR THE EXPERIMENT

\begin{tabular}{|lll|lll|}
\hline$M_{m}$ & 5.83 & {$[\mathrm{~kg}]$} & $M_{s}$ & 0.019 & {$[\mathrm{~kg}]$} \\
$B_{m}$ & 13.3 & {$[\mathrm{Ns} / \mathrm{m}]$} & $B_{s}$ & 1.158 & {$[\mathrm{Ns} / \mathrm{m}]$} \\
\hline
\end{tabular}

and the differentiated position signal. The slave is a one dimensional system, shown in Fig.9, with linear voice-coil actuator, span of $12 \mathrm{~mm}$, a linear encoder with resolution of $0.5 \mu \mathrm{m}$ and a force sensor that has a resolution of $0.3 \mathrm{mg}$, but only accuracy of about $2 \mathrm{~g}$ over a range of $20 \mathrm{~g}$.

Considering the hardware limitations, we set the range of scaling as $5 \leq \phi \leq 10$ and $25 \leq \varepsilon \leq 100$. Experiments were done where contact with soft and rigid environments were made. During the experiment the scale factors were adapted by the user who turned two dials (Fig.10). The identified parameters of the master and slave arms are given in Table I. Weightings in $\boldsymbol{W}$ are set as shown in Table II, where the gains of $W_{p}$ and $W_{f}$ are made scale-dependent. The weighting parameter $\rho$ in (11) was set to 50 .

\section{B. Experimental results}

Figure 11 shows the experimental results for the interaction with a spongy environment. The graphs on the left show the position signals, the variation of the position scale factor, the force signals and lastly the variation of the force scale factor. The graph on the right shows the variation of the scale

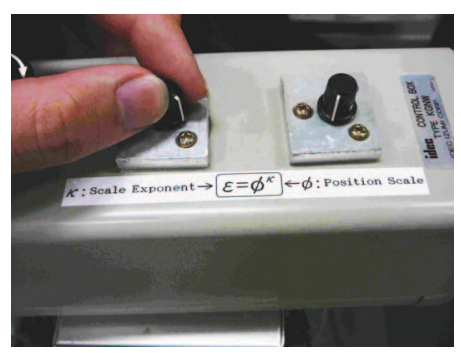

Fig. 10. Scale adjustment dials 

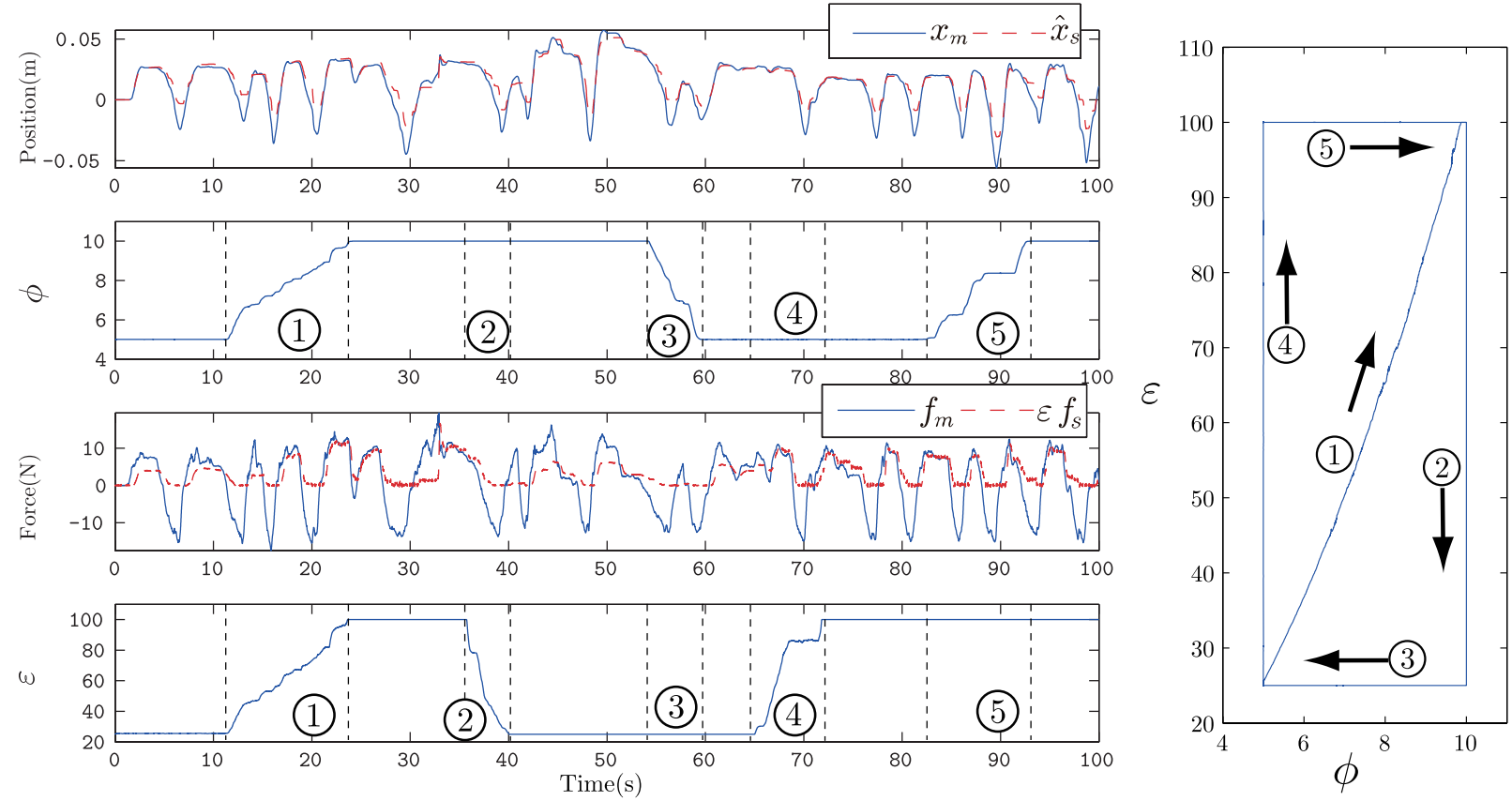

(a) positions, forces and scale factors

(b) locus of the selected scales

Fig. 11. Experimental result (Interaction with a sponge)

TABLE II

WEIGHTING FUNCTIONS FOR CONTROL OBJECTIVES $\left(\times 10^{3}\right)$

\begin{tabular}{|l|llll|}
\hline & $W_{p}$ & $W_{f}$ & $W_{u m}$ & $W_{u s}$ \\
\cline { 2 - 5 } & $\frac{K_{p}(\boldsymbol{\alpha})}{s+10}$ & $\frac{K_{f}(\boldsymbol{\alpha})}{s+10}$ & $\frac{K_{u m}(s+100)}{s+1000}$ & $\frac{K_{u s}(s+100)}{s+1000}$ \\
$\operatorname{vertex}(\phi, \varepsilon)$ & $K_{p}$ & $K_{f}$ & $K_{u m}$ & $K_{u s}$ \\
\hline $\boldsymbol{\alpha}_{1}(5,25)$ & 96 & 16.0 & 3 & 2.5 \\
$\boldsymbol{\alpha}_{2}(10,25)$ & 32 & 22.4 & 3 & 2.5 \\
$\boldsymbol{\alpha}_{3}(5,100)$ & 224 & 3.2 & 3 & 2.5 \\
$\boldsymbol{\alpha}_{4}(10,100)$ & 160 & 9.6 & 3 & 2.5 \\
\hline
\end{tabular}

factor over the parameter space.

Each time the master signals are plotted together with the scaled slave signals. It can be seen that these lines correspond well, confirming the validity of the proposed methods. The relative low accuracy of the force sensor of the slave, urged us to set force gains lower than positional gains, reflecting in the somewhat lower quality of the force sensing.

Stable behavior over the whole parameter space was verified, both for independent variations of $\varepsilon(t)$ and $\phi(t)$ (as in (2), (3), (4) and (5)) as in dependent variations, e.g. along the line (1). Note, that the line (1) is of practical interest, as here the relation $\varepsilon(t) \approx \phi^{2}(t)$ holds. Practically, this combination of scale factors will correctly scale the spring forces of the remote environment [4]. Also a stable contact with a rigid environment (metal stopper) was obtained.

\section{CONCLUSIONS AND FURTHER WORKS}

This paper discussed variable-scale bilateral control for micro teleoperation. First, we formulated the scaled teleoperation system interacting with a passive operator and environment as a generalized plant with a structured uncertainty. Then, it was shown how robust performant controllers for scaled teleoperation can be calculated by solving a set of LMI's, augmented with some nonconvex rank conditions.

Next, it was found that the scaled teleoperation robust performance problem has an affine dependence on the scale factors. The generalized plant of scaled teleoperation is therefore a polytopic linear varying plant, for which efficient gain-scheduling methods exist.

To improve the performance of the gain-scheduled controllers, some of the weightings of the control objectives are made a function of the schedule parameters. A simple heuristic was proposed to maintain the polytopic nature of the weighted plant. Further work should be done to investigate if smarter choices of scale factor dependence of the weightings can improve the performance even further.

Experimental results confirmed the validity of the proposed approach. The system was kept stable under contacts with a spongy as well as a rigid environment, while velocity and/or force scaling factors varied.

For future works, the ranges of the scaling factor should be set wider to get more benefit from the scale-dependent weightings. To do so, a more sophisticated sensor and actuator at the slave should be constructed. Extension to multiple d.o.f. and integration of the system with an intuitive camera guidance system should be performed as well. Extension of the proposed framework to deal with master and slave modeling errors should be trivial, but also uncertainty caused by time delay must be dealt with. 


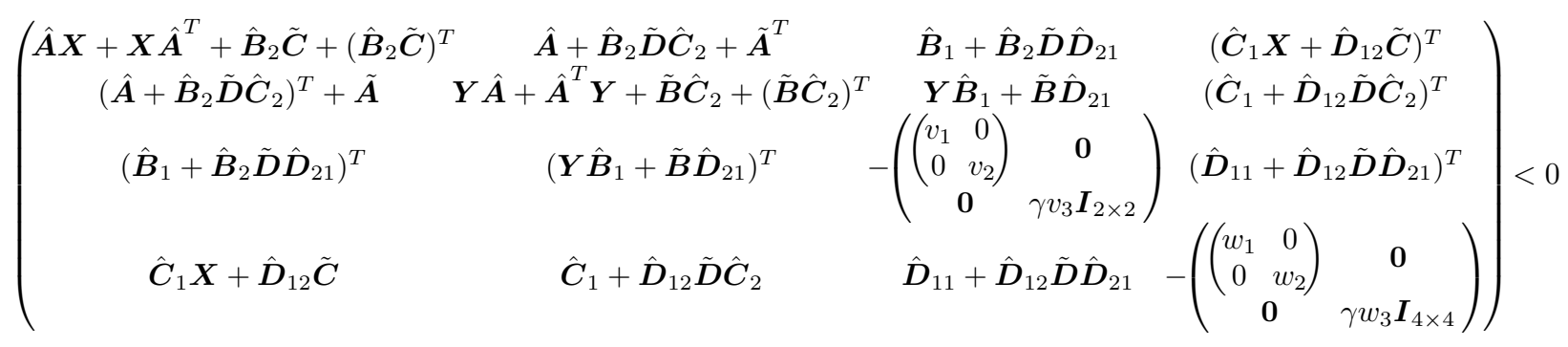

\section{APPENDIX}

\section{A. Synthesis of constant scaled $\mathcal{H}_{\infty}$ controllers by LMI's}

The $\mathcal{H}_{\infty}$ constraint, described by (18), is now reformulated as a set of LMI's, augmented with some matrix rank conditions. For a detailed procedure, please refer to [13]. Here, we limit us to say that after applying the Bounded Real Lemma [21] on (18) and after performing two Schur complements, (18) can be reformulated [22] as the algebraic Riccati inequality (ARI) of (25), under constraints

$$
v_{i}=w_{i}^{-1}>0 \quad(i=1,2) .
$$

A positive definite solution of the ARI exists if (25) and

$$
\left[\begin{array}{cc}
\boldsymbol{X} & \boldsymbol{I}_{8 \times 8} \\
\boldsymbol{I}_{8 \times 8} & \boldsymbol{Y}
\end{array}\right]>0
$$

hold. In this paper the order of the controllers are the same as the order of the weighted plant. For a plant with 4 states and first order weights $W_{p}, W_{f}, W_{u m}, W_{u s}$, this gives controllers of order 8. A suboptimal controller is then found by

$$
v_{3} w_{3}=\gamma^{2} \rightarrow \text { minimal. }
$$

Note that (25) is affine in the variables $X, Y, \tilde{A}, \tilde{B}, \tilde{C}, \tilde{D}$, which are defined as:

$$
\begin{aligned}
& \tilde{\boldsymbol{A}}=\boldsymbol{N} \boldsymbol{A}_{K} \boldsymbol{M}^{T}+\boldsymbol{N B}_{K} \hat{\boldsymbol{C}}_{2} \boldsymbol{X}+\boldsymbol{Y} \hat{\boldsymbol{B}}_{2} \boldsymbol{C}_{K} \boldsymbol{M}^{T}+\boldsymbol{Y}\left(\hat{\boldsymbol{A}}+\hat{\boldsymbol{B}}_{2} \boldsymbol{D}_{K} \hat{\boldsymbol{C}}_{2}\right) \boldsymbol{X}, \\
& \tilde{\boldsymbol{B}}=\boldsymbol{N} \boldsymbol{B}_{K}+\boldsymbol{Y} \hat{\boldsymbol{B}}_{2} \boldsymbol{D}_{K}, \tilde{\boldsymbol{C}}=\boldsymbol{C}_{K} \boldsymbol{M}^{T}+\boldsymbol{D}_{K} \hat{\boldsymbol{C}}_{2} \boldsymbol{X}, \tilde{\boldsymbol{D}}=\boldsymbol{D}_{K},
\end{aligned}
$$

for $\boldsymbol{N} \boldsymbol{M}^{T}=\boldsymbol{I}_{8 \times 8}-\boldsymbol{Y} \boldsymbol{X}<0$. The constraints (26) can be reformulated [23] as:

$$
\left[\begin{array}{cc}
v_{i} & 1 \\
1 & w_{i}
\end{array}\right] \geq 0
$$

and

$$
\operatorname{rank}\left(\left[\begin{array}{cc}
v_{i} & 1 \\
1 & w_{i}
\end{array}\right]\right) \leq 1,(i=1,2) .
$$

The solution to the constant scaled $\mathcal{H}_{\infty}$ problem can be found by searching for the solution to the LMI's (25), (27) and (30), under rank condition (31), that minimizes (28).

After finding $\boldsymbol{X}, \boldsymbol{Y}, \tilde{\boldsymbol{A}}, \tilde{\boldsymbol{B}}, \tilde{\boldsymbol{C}}, \tilde{\boldsymbol{D}}$, the state space matrices of the controller: $\boldsymbol{A}_{K}, \boldsymbol{B}_{K}, \boldsymbol{C}_{K}$ and $\boldsymbol{D}_{K}$ can be found by solving (29) for a certain choice of $\boldsymbol{M}$ and $\boldsymbol{N}$.

\section{REFERENCES}

[1] J. Hightower and D. Smith, "Teleoperator technology development," in Proceedings, 12th Meeting of UJNR/MFP, 1983, pp. 43-47.

[2] S. Tachi and K. Yasuda, "Evaluation experiments of a teleexistence manipulation system," Presence, vol. 3, no. 1, pp. 35-44, 1994.
[3] J. E. Colgate, "Robust impedance shaping telemanipulation," IEEE Transactions on robotics and automation, vol. 9, no. 4, pp. 374-384, August 1993.

[4] Y. Yokokohji, N. Hosotani, J. Ueda, and T. Yoshikawa, "A micro teleoperation system for compensating scaling effects based on environment model," in Japan-USA Symposium on Flexible Automation, Kobe, Japan, 1994, pp. 709-716.

[5] K. Kosuge, T. Itoh, and T. Fukuda, "Scaled telemanipulation with communication time delay," in Proc. IEEE Int. Conf. on Robotics and Automation, Mineapolis, Minnesota, April 1996, pp. 2019-2024.

[6] R. Dubey, S. Everett, N. Pernalette, and K. Manocha, "Teleoperator assistance through variable velocity mapping," IEEE Transactions on Robotics and Automation, vol. 17, no. 5, Oct. 2001

[7] M. Boukhnifer, A. Ferreira, and J. Fontain, "Scaled teleoperation controller design for micromanipulation over internet," in Proc. IEEE Int. Conf. on Robotics and Automation, Apr. 2004, pp. 4577-4583.

[8] A. Sano, H. Fujimoto, and T. Takai, "Human-centered scaling in micro-teleoperation," in Proc. IEEE Int. Conf. on Robotics and Automation, Seoul, May 2001, pp. 380-385.

[9] —-, "Network-based force-reflecting teleoperation," in Proc. IEEE Int. Conf. on Robotics and Automation, San Francisco, CA, April 2000, pp. 3126-3131.

[10] N. Hogan, "Controlling impedance at the man/machine interface," in Proc. of the 1989 IEEE International Conference on Robotics and Automation (Vol. 3), Scottsdale, AZ, 1989, pp. 1626-1631.

[11] J. Doyle, "Analysis of feedback systems with structured uncertainties," IEE Proceedings, vol. 129, Part D, no. 6, pp. 242-250, Nov. 1982.

[12] T. Yoshikawa, Y. Yokokohji, N. Hosotani, and J.-i. Imura, "Robust control of master-slave systems considering uncertainties of environment and operator," Journal of Robotics Society of Japan, vol. 14 no. 6, pp. 836-845, 1996, (in Japanese).

[13] E. Vander Poorten, "Improving haptic fidelity for intuitive manipulation in scaled teleoperation and virtual environment," $\mathrm{Ph} . \mathrm{D}$. dissertation, Kyoto University, april 2007.

[14] P. Gahinet, A. Nemirovski, A. Laub, and M. Chilali, LMI Control Toolbox, For Use with MATLAB. The MathWorks, Inc., 1995.

[15] L. Ghaoui, F. Oustry, and M. Rami, "A cone complementarity linearization algorithm for static output feedback and related problems," IEEE Transactions on Automatic Control, vol. 42, no. 8, pp. 1171$1176,1997$.

[16] T. Iwasaki and R. Skelton, "The xy-centering algorithm for the dual lmi problem: A new approach to fixed order control design," INT. Journal of Control, vol. 62, no. 6, pp. 1257-1272, 1995.

[17] K. Grigoriadis and R. Skelton, "Low-order control design for 1mi problems using alternating projection methods," Automatica, vol. 32, no. 8 , pp. $1117-1125,1996$.

[18] J. Shamma and M. Athans, "Guaranteed properties of gain scheduled control for linear parameter-varying plants," Automatica, vol. 27, no. 3, pp. 559-564, 1991.

[19] P. Apkarian, P. Gahinet, and B. Greg, "Self-scheduled $\mathcal{H}_{\infty}$ control of linear parameter-varying systems: a design example," Automatica, vol. 31, no. 9, pp. 1251-1261, 1995.

[20] W. Yang and P. Becker, "Induced $l_{2}$-norm control for LPV systems with bounded parameter variation," Int. J. Robust Nonlinear Control, vol. 6, no. 9/10, pp. 983-998, 1996. [Online]. Available: citeseer.ist.psu.edu/yang96induced.html

[21] P. Gahinet and P. Apkarian, "A linear matrix inequality approach to $\mathcal{H}_{\infty}$ control," Int. Journal of Robust and Nonlinear Control, pp. 421448, 1994.

[22] A. Fujimori, Robust Control. Coron, 2001 (in Japanese).

[23] T. Iwasaki, LMI and Control. Shokodo, 1997 (in Japanese). 